\title{
Dynamics Control by a Time-varying Feedback
}

\author{
A.A. Agrachev M. Caponigro
}

July 24, 2009

\begin{abstract}
We consider a smooth bracket generating control-affine system in $\mathbb{R}^{d}$ and show that any orientation preserving diffeomorphism of $\mathbb{R}^{d}$ can be approximated, in the very strong sense, by a diffeomorphism included in the flow generated by a time-varying feedback control which is polynomial with respect to the state variables and trigonometricpolynomial with respect to the time variable.
\end{abstract}

\section{Introduction}

We consider a control-affine system:

$$
\dot{q}=f_{0}(q)+\sum_{i=1}^{m} u_{i} f_{i}(q), \quad q \in \mathbb{R}^{d},
$$

with $u=\left(u_{1}, \ldots, u_{m}\right) \in \mathbb{R}^{m}$, where $f_{i}$ are smooth (i.e. $\left.C^{\infty}\right)$ vector fields on $\mathbb{R}^{d}$. Moreover, we assume that $\left\{f_{1}, \ldots, f_{m}\right\}$ is a bracket generating family of vector fields, i.e. $\operatorname{Lie}_{q}\left\{f_{1}, \ldots, f_{m}\right\}=\mathbb{R}^{d}$, for any $q \in \mathbb{R}^{d}$, where $\operatorname{Lie}_{q}\left\{f_{1}, \ldots, f_{m}\right\}$ is the linear hull of all iterated Lie brackets of the fields $f_{1}, \ldots, f_{m}$ evaluated at $q$.

Feedback control (or time-invariant feedback control) is a mapping

$$
v=\left(v_{1}, \ldots, v_{m}\right): \mathbb{R}^{d} \rightarrow \mathbb{R}^{m} .
$$

We can put $u_{i}=v_{i}(q)$ and obtain a closed loop system

$$
\dot{q}=f_{0}(q)+\sum_{i=1}^{m} v_{i}(q) f_{i}(q), \quad q \in \mathbb{R}^{d} .
$$


It is very interesting to know what kind of dynamics we can realize by an appropriate choice of the feedback control. Of course, a smooth or at least Lipschitz feedback is preferable if we want system (2) to correctly define a dynamical system. Unfortunately, we cannot expect too much. In particular, if $f_{0}=0$ then system (2) with a continuous feedback control cannot have locally asymptotically stable equilibria as it was observed by R. Brockett [2].

J.-M. Coron suggested to use time-varying periodic with respect to time feedback controls

$$
v: \mathbb{R} \times \mathbb{R}^{d} \rightarrow \mathbb{R}^{d}, \quad v(t+1, q)=v(t, q), \quad q \in \mathbb{R}^{d}, t \in \mathbb{R},
$$

for system (1) and proved that asymptotic stability can be successfully achieved by a $C^{\infty}$ time-varying feedback (see $[4,5]$ or $[6$, section 11.2]).

In this paper, we focus on the transformation $q(0) \mapsto q(1)$ in virtue of the system

$$
\dot{q}=f_{0}(q)+\sum_{i=1}^{m} v_{i}(t, q) f_{i}(q), \quad q \in \mathbb{R}^{d} .
$$

associated to the time-varying feedback control and demonstrate that practically any type of discrete-time dynamics can be realized in this way.

More precisely, let $\Phi_{v}: q(0) \mapsto q(1)$ be the transformation of $\mathbb{R}^{d}$ which sends the initial value of any solution of system (3) to its value at $t=1$. We denote by Diff $\mathbb{R}^{d}$ the group of orientation preserving diffeomorphisms of $\mathbb{R}^{d}$. Let $P \in \operatorname{Diff}_{0} \mathbb{R}^{d}, \mathcal{O}_{P}$ be a $C^{\infty}$-neighborhood of $P$ and $N$ be a positive integer. We prove (Theorem 8) that there exists a polynomial with respect to $q$ and trigonometric polynomial with respect to $t$ time-varying feedback control $v$ such that $\Phi_{v} \in \mathcal{O}_{P}$ and the $N$-jets of $\Phi_{v}$ and $P$ at the origin coincide. Moreover, construction of the time-varying feedback $v$ is surprisingly simple.

Let us fix some notations. We denote by $\operatorname{Diff}\left(\mathbb{R}^{d}\right)$ the group of diffeomorphisms of $\mathbb{R}^{d}$ and by Vec $\mathbb{R}^{d}$ the space of vector fields on $\mathbb{R}^{d}$. We assume that $\operatorname{Diff}\left(\mathbb{R}^{d}\right), \operatorname{Diff} 0\left(\mathbb{R}^{d}\right), \operatorname{Vec} \mathbb{R}^{d}$, and $C^{\infty}\left(\mathbb{R}^{d}\right)$ are endowed with the standard topology of the uniform convergence of the partial derivatives of any order on any compact of $\mathbb{R}^{d}$.

Given a set $\mathcal{F}$ of vector fields on $\mathbb{R}^{d}$, we denote by:

$$
\operatorname{Gr} \mathcal{F}=\left\{e^{t_{1} f_{1}} \circ \cdots \circ e^{t_{k} f_{k}} \mid t_{i} \in \mathbb{R}, f_{i} \in \mathcal{F}, k \in \mathbb{N}\right\},
$$

the subgroup of Diff $\mathbb{R}^{d}$ generated by flows of vector fields in $\mathcal{F}$, and by:

$$
\mathrm{Gr}_{S} \mathcal{F}=\left\{e^{a_{1} f_{1}} \circ \cdots \circ e^{a_{k} f_{k}} \mid a_{i} \in C^{\infty}\left(\mathbb{R}^{d}\right), f_{i} \in \mathcal{F}, k \in \mathbb{N}\right\},
$$


respectively the subgroup of Diff $\mathbb{R}^{d}$ generated by flows of vector fields in $\mathcal{F}$ rescaled by smooth functions on $\mathbb{R}^{d}$. We consider time-varying vector fields $V_{t}(q)$ on $\mathbb{R}^{d}$ that are smooth with respect to $q \in \mathbb{R}^{d}$ and locally integrable with respect to $t \in \mathbb{R}$. All vector fields under consideration are supposed to satisfy the growth condition $V_{t}(q) \leq \varphi(t)(1+|q|)$, where $\varphi$ is a locally integrable function. This condition guarantees completeness of the vector field.

Given a time-varying vector field $V_{t}(q)$ on $\mathbb{R}^{d}$, let

$$
P_{t}: \mathbb{R}^{d} \rightarrow \mathbb{R}^{d}, \quad t \in \mathbb{R}
$$

be the (non-stationary) flow generated by the differential equation $\dot{q}=V_{t}(q)$. In other words,

$$
\left\{\begin{array}{l}
\frac{\partial P_{t}}{\partial t}(q)=V_{t}\left(P_{t}(q)\right), \\
P_{0}(q) \equiv q .
\end{array}\right.
$$

In the following we will use the "chronological" notation: $P_{t}=\overrightarrow{\exp } \int_{0}^{t} V_{\tau} d \tau$, to denote such a flow.

Recall that if $\mathcal{F}$ is a bracket generating family of vector fields then by Rashevski-Chow Theorem (see $[8,3]$ ), for every $q_{0}$, the orbit $\mathcal{O}_{q_{0}}$ of the family is the whole space $\mathbb{R}^{d}$ moreover, according to the Orbit Theorem of Sussmann([9] or [1, chapter 5]), any smooth vector field can be presented as a linear combination of vector fields from $\mathcal{F}$ transformed by diffeomorphisms from $\operatorname{Gr} \mathcal{F}$. In particular it is possible to take $X_{1}, \ldots, X_{d}$ linearly independent at a point $q \in \mathbb{R}^{d}$ and such that $X_{i}=P^{i}{ }_{*} f_{i}, i=1, \ldots, d$ with $P^{i} \in$ Gr $\mathcal{F}$ and $f_{i} \in \mathcal{F}$.

The main result proved in this paper is as follows.

Theorem. Let $\left\{f_{1}, f_{2}, \ldots, f_{m}\right\}$ be a bracket generating family of vector fields on $\mathbb{R}^{d}$. Consider the control system

$$
\dot{q}=f_{0}(q)+\sum_{i=1}^{m} u_{i}(t, q) f_{i}(q), \quad q \in \mathbb{R}^{d},
$$

with controls $u_{i}$ such that:

(i) $u_{i}$ is polynomial with respect to $q \in \mathbb{R}^{d}$;

(ii) $u_{i}$ is a trigonometric polynomial with respect to $t \in[0,1]$; 
for every $i=1, \ldots, m$.

Fix positive integers $N$ and $k, \varepsilon>0$, and $B$ ball in $\mathbb{R}^{d}$. For any $\Phi \in$ $\operatorname{Diff}_{0}\left(\mathbb{R}^{d}\right)$, there exist controls $u_{1}(t, q), \ldots, u_{m}(t, q)$ such that, if $P$ is the flow at time 1 of the system, then

$$
J_{0}^{N}(P)=J_{0}^{N}(\Phi) \quad \text { and } \quad\|P-\Phi\|_{C^{k}(B)}<\varepsilon .
$$

Proof is divided into four sections. In Section 2 we consider a bracket generating family of vector fields closed under multiplication by smooth functions on $\mathbb{R}^{d}$, say $\mathcal{F}$, then we prove that the group of diffeomorphisms generated by flows of vector fields in this family is dense in the connected component of the identity of the group of diffeomorphisms. In Section 3 we use classical Implicit Function Theorem to prove that the $N$-th jet of a diffeomorphism in $\operatorname{Diff}_{0}\left(\mathbb{R}^{d}\right)$ sufficiently close to the identity can be presented as the $N$-th jet of an element in Gr $\mathcal{F}$. Then using Proposition 2 we can extend this result to every diffeomorphism in $\operatorname{Diff}_{0}\left(\mathbb{R}^{d}\right)$. The results of Section 2 and Section 3 are combined together in Section 4 to prove that it is possible to find an element in the group $\mathrm{Gr} \mathcal{F}$ with the same $N$-th jet of a given diffeomorphism and also close to it in the $C^{\infty}$-topology. This result, as showed in Section 5, implies the main result in the driftless case, namely $f_{0} \equiv 0$, and with controls $u_{i}(t, \cdot)$ that are piecewise constant with respect to $t$. Therefore we use Brouwer Fixed Point to prove that it is possible to perturb the map

$$
\left(u_{1}, \ldots, u_{m}\right) \mapsto J_{0}^{N}\left(\overrightarrow{\exp } \int_{0}^{1} \sum_{i=1}^{m} u_{i}(t, \cdot) f_{i}(\cdot) d t\right)
$$

without losing surjectivity. This argument leads to the proof of the Theorem.

\section{An approximation result}

We start with a simple modification of a standard relaxation result (see [1, chapter 8] or [7]). Its proof is done in the appendix for convenience of the reader.

Proposition 1. Let $X_{1}, \ldots, X_{k}$ be smooth vector fields on $\mathbb{R}^{d}$ and $\mathcal{A}$ be a closed subspace of $C^{\infty}\left(\mathbb{R}^{d}\right)$. Then, for any time-varying vector field of the form

$$
V_{t}=\sum_{i=1}^{k} a_{i}(t, \cdot) X_{i}
$$


where $a_{i}(t, \cdot) \in \mathcal{A}$ and $0 \leq a_{i}(t, q) \leq \varphi(t)$ for some locally integrable $\varphi, i=$ $1, \ldots, k$, there exists a sequence of time-varying, piecewise constant with respect to $t$, vector fields $Z_{t}^{n}$ such that

$$
Z_{t}^{n} \in\left\{a X_{i} \mid a \in \mathcal{A}, i=1, \ldots, k\right\}, \quad \text { for any } t \in[0,1]
$$

and

$$
\overrightarrow{\exp } \int_{0}^{t} Z_{\tau}^{n} d \tau \longrightarrow \overrightarrow{\exp } \int_{0}^{t} V_{\tau} d \tau, \quad \text { as } n \rightarrow \infty
$$

in the standard topology and uniformly with respect to $t \in[0,1]$.

Proposition 2 (Approximation). Let $\mathcal{F} \subseteq$ Vec $\mathbb{R}^{d}$ be a bracket generating family of vector fields on $\mathbb{R}^{d}$ such that

$$
\text { af } \in \mathcal{F} \text { for any } a \in C^{\infty}\left(\mathbb{R}^{d}\right), f \in \mathcal{F} \text {. }
$$

Then, for any orientation preserving diffeomorphism $P$ of $\mathbb{R}^{d}$, there exists a sequence $\left\{P_{n}\right\}_{n} \subset \mathrm{Gr} \mathcal{F}$ such that

$$
P_{n} \longrightarrow P, \quad \text { as } n \rightarrow \infty
$$

in the standard topology.

Proof. First, note that any orientation preserving diffeomorphism of $\mathbb{R}^{d}$ is isotopic to the identity. Indeed, let $P$ be an orientation preserving diffeomorphisms of $\mathbb{R}^{d}$. We can suppose without loss of generality that $P$ fixes the origin just taking the isotopy $H^{1}(t, \cdot)=P-(1-t) P(0)$. Now, rename for simplicity $P:=H^{1}(0, \cdot)$ and consider another isotopy

$$
H^{2}(t, q)=P(t q) / t, \quad t \in(0,1], \quad \text { and } \quad H^{2}(0, q)=\lim _{t \rightarrow 0} P(t q) / t .
$$

Since $P$ is orientation preserving then $H^{2}(0, \cdot)$ belongs to the connected component of the identity of the group of linear invertible operators on $\mathbb{R}^{d}$, $G L^{+}(d, \mathbb{R})$.

Let $P^{t} \subset \operatorname{Diff}_{0}\left(\mathbb{R}^{d}\right)$ be a path such that $P^{0}=$ Id and $P^{1}=P$. Consider the time-varying vector field

$$
V_{t}=\left(P^{t}\right)^{-1} \circ \frac{d}{d t} P^{t}
$$


We have

$$
\overrightarrow{\exp } \int_{0}^{t} V_{\tau} d \tau=P^{t}
$$

Recall that, since $\mathcal{F}$ is bracket generating then it is possible to take $X_{1}, \ldots, X_{d}$ such that $X_{i}=P_{*}^{i} f_{i}$ with $P^{i} \in \operatorname{Gr} \mathcal{F}, f_{i} \in \mathcal{F}, i=1, \ldots, d$ and

$$
V_{t}=\sum_{i=1}^{d} a_{i}(t, \cdot) X_{i}
$$

where $a_{i}(t, \cdot) \in C^{\infty}\left(\mathbb{R}^{d}\right)$ for any $t \in[0,1]$.

By Proposition 1 there exists a sequence $Z_{t}^{n} \in\left\{\alpha X_{i} \mid \alpha \in C^{\infty}\left(\mathbb{R}^{d}\right), i=\right.$ $1, \ldots, d\}$ such that

$$
\overrightarrow{\exp } \int_{0}^{t} Z_{\tau}^{n} d \tau \rightarrow P^{t}, \quad \text { as } n \rightarrow \infty
$$

and the convergence is uniform with respect to $t \in[0,1]$. Let $P_{n}:=\overrightarrow{\exp } \int_{0}^{1} Z_{t}^{n} d t$, then

$$
P_{n} \rightarrow P, \quad \text { as } n \rightarrow \infty .
$$

It remains to prove that $P_{n} \in \operatorname{Gr} \mathcal{F}$ for every $n$. Since $Z_{t}^{n}$ is piecewise constant in $t$, so, for any fixed $n \in \mathbb{N}$, there exist disjoint segments $I_{1}, \ldots, I_{h_{n}}$ covering $[0,1]$ and functions $\alpha_{1}, \ldots, \alpha_{h_{n}} \in C^{\infty}\left(\mathbb{R}^{d}\right)$ such that

$$
Z_{t}^{n}=\alpha_{k} X_{i_{k}} \quad \forall t \in I_{k}, \quad k=1, \ldots, h_{n} .
$$

Hence

$$
\begin{aligned}
P_{n} & =\overrightarrow{\exp } \int_{0}^{1} Z_{t}^{n} d t \\
& =\quad e^{\left|I_{1}\right| \alpha_{1} X_{i_{1}}} \circ \cdots \circ e^{\left|I_{h_{n}}\right| \alpha_{h_{n}} X_{h_{h_{n}}}} \\
& =\quad e^{\left|I_{1}\right| \alpha_{1} P_{*}^{i_{1}} f_{i_{1}}} \circ \cdots \circ e^{\left|I_{h}\right| \alpha_{h} P_{*}^{i_{h_{n}}} f_{i_{h_{n}}}} \\
& =\quad\left(P^{i_{1}}\right)^{-1} \circ e^{\left|I_{1}\right|\left(\alpha_{1} \circ P^{i_{1}}\right) f_{i_{1}}} \circ P^{i_{1}} \circ \cdots \\
& \circ\left(P^{i_{h_{n}}}\right)^{-1} \circ e^{\left|I_{h}\right|\left(\alpha_{h_{n}} \circ P^{i_{h_{n}}}\right) f_{i_{h_{n}}} \circ P^{i_{h_{n}}},}
\end{aligned}
$$


now let $\beta_{k}=\left|I_{k}\right|\left(\alpha_{k} \circ P^{i_{k}}\right)$, then

$$
P_{n}=\left(P^{i_{1}}\right)^{-1} \circ e^{\beta_{1} f_{i_{1}}} \circ P^{i_{1}} \circ \cdots \circ\left(P^{i_{h_{n}}}\right)^{-1} \circ e^{\beta_{h_{n}} f_{i_{h_{n}}}} \circ P^{i_{h_{n}}},
$$

and $P_{n} \in \mathrm{Gr} \mathcal{F}$ by assumption (5).

In other words we proved that if $\mathcal{F}$ is a bracket generating family of vector fields then $\mathrm{Gr}_{S} \mathcal{F}$ is dense in the connected component of the identity of Diff $\mathbb{R}^{d}$ endowed with the standard $C^{\infty}$-topology.

\section{Get the jet}

In this section, given a bracket generating family of vector fields $\mathcal{F}$, we find a diffeomorphism in the group $\mathrm{Gr}_{S} \mathcal{F}$ whose $N$-th jet is exactly the $N$-th jet of a given diffeomorphism on $\mathbb{R}^{d}$. The main tool used is the classical Implicit Function Theorem.

Proposition 3. Let $\mathcal{F}$ be a bracket generating family of vector fields on $\mathbb{R}^{d}$ and $N>0$ a positive integer.

For any diffeomorphism $\Phi: \mathbb{R}^{d} \rightarrow \mathbb{R}^{d}$ sufficiently close to the identity there exists $P \in \mathrm{Gr}_{S} \mathcal{F}$ such that

$$
J_{0}^{N}(P)=J_{0}^{N}(\Phi)
$$

Proof. Consider a frame of vector fields linearly independent in $0 \in \mathbb{R}^{d}$, $X_{1}, \ldots, X_{d}$. Let $\mathbf{X}$ be the space of polynomials of degree less or equal than $N$ in $d$ variables and let $\mathbf{Y}$ be the jet-group of $N$-th order jets at 0 of smooth orientation preserving diffeomorphisms, i.e. $\mathbf{Y}=J_{0}^{N}\left(\operatorname{Diff}_{0}\left(\mathbb{R}^{d}\right)\right)$. Note that $\operatorname{dim} \mathbf{X}<\infty$ and $\operatorname{dim} \mathbf{Y}<\infty$.

Consider the map

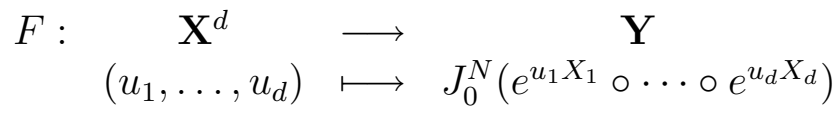

We want to prove that Implicit Function Theorem applies. Let us compute the differential of $F$ at $0 \in \mathbf{X}^{d}$

$$
\begin{aligned}
D_{0} F\left(a_{1}, \ldots, a_{d}\right) & =\left.\frac{\partial F}{\partial u_{1}}\right|_{u_{1}=\ldots=u_{d}=0} a_{1}+\cdots+\left.\frac{\partial F}{\partial u_{d}}\right|_{u_{1}=\ldots=u_{d}=0} a_{d} \\
& =a_{1} J_{0}^{N}\left(X_{1}\right)+\cdots+a_{d} J_{0}^{N}\left(X_{d}\right) .
\end{aligned}
$$


We claim that $D_{0} F: \mathbf{X}^{d} \rightarrow T_{\text {Id }} \mathbf{Y}$ is invertible. In fact

$$
T_{\mathrm{Id}} \mathbf{Y}=T_{\mathrm{Id}} J_{0}^{N}\left(\operatorname{Diff}_{0}\left(\mathbb{R}^{d}\right)\right)=J_{0}^{N}\left(T_{\mathrm{Id}} \operatorname{Diff}_{0}\left(\mathbb{R}^{d}\right)\right)=J_{0}^{N}\left(\operatorname{Vec}\left(\mathbb{R}^{d}\right)\right),
$$

so for every $V \in J_{0}^{N}\left(\operatorname{Vec}\left(\mathbb{R}^{d}\right)\right)$ there exist $b_{1}, \ldots, b_{d}$ such that

$$
\begin{aligned}
V & =J_{0}^{N}\left(b_{1} X_{1}+\cdots+b_{d} X_{d}\right) \\
& =J_{0}^{N}\left(b_{1}\right) J_{0}^{N}\left(X_{1}\right)+\ldots+J_{0}^{N}\left(b_{d}\right) J_{0}^{N}\left(X_{d}\right) .
\end{aligned}
$$

Every element $V \in T_{\mathrm{Id}} \mathbf{Y}$ is image of $d$ polynomials of degree less or equal than $N, a_{i}=J_{0}^{N}\left(b_{i}\right)$. Therefore there exists $\mathcal{O}$ neighborhood of Id in $\mathbf{Y}$ such that $F$ is locally surjective on $\mathcal{O}$. Namely, for every $\psi \in \mathcal{O}$, there exist $\left(u_{1}, \ldots, u_{d}\right) \in \mathbf{X}^{d}$ such that $F\left(u_{1}, \ldots, u_{d}\right)=\psi$.

If $\Phi$ is sufficiently close to the identity, then $J_{0}^{N}(\Phi) \in \mathcal{O}$. Therefore there exist polynomials $v_{1}, \ldots, v_{d} \in \mathbf{X}^{d}$ such that

$$
J_{0}^{N}\left(e^{v_{1} X_{1}} \circ \cdots \circ e^{v_{d} X_{d}}\right)=J_{0}^{N}(\Phi) .
$$

It remains to prove that $P=e^{v_{1} X_{1}} \circ \cdots \circ e^{v_{d} X_{d}} \in \mathrm{Gr}_{S} \mathcal{F}$, but according to Orbit Theorem, for $i=1, \ldots, d$, we have that $X_{i}=P_{*}^{i} f_{i}$, where $f_{i} \in \mathcal{F}$ and $P^{i} \in \operatorname{Gr} \mathcal{F}$. Let $P^{i}=e^{t_{1}^{i} f_{1}^{i}} \circ e^{t_{2}^{i} f_{2}^{i}} \circ \cdots \circ e^{t_{s_{i}}^{i} f_{s_{i}}^{i}}$ with $f_{j}^{i} \in \mathcal{F}$. Therefore

$$
\begin{aligned}
& P=e^{v_{1} P_{*}^{1} f_{1}} \circ \cdots \circ e^{v_{d} P_{*}^{d} f_{d}} \\
& =P^{1} \circ e^{\left(P^{1}\right)^{-1}\left(v_{1}\right) f_{1}} \circ\left(P^{1}\right)^{-1} \circ \cdots \circ P^{d} \circ e^{\left(P^{d}\right)^{-1}\left(v_{d}\right) f_{d}} \circ\left(P^{d}\right)^{-1}
\end{aligned}
$$

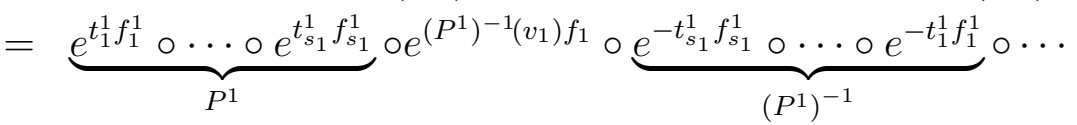

$$
\begin{aligned}
& \circ \underbrace{e^{t_{1}^{d} f_{1}^{d}} \circ \cdots \circ e^{t_{s_{d}}^{d} f_{s_{d}}^{d}}}_{P^{d}} \circ e^{\left(P^{d}\right)^{-1}\left(v_{d}\right) f_{d}} \circ \underbrace{e^{-t_{s_{d}}^{d} f_{s_{d}}^{d}} \circ \cdots \circ e^{-t_{1}^{d} f_{1}^{d}}}_{\left(P^{d}\right)^{-1}} \\
& =e^{w_{1} g_{1}} \circ \cdots \circ e^{w_{\ell} g_{\ell}},
\end{aligned}
$$

with $g_{1}, \ldots, g_{\ell} \in \mathcal{F}$ and $\ell=d+2\left(s_{1}+\cdots+s_{d}\right)$. Therefore $P \in \operatorname{Gr}_{S} \mathcal{F}$ and the proposition follows.

Now consider any diffeomorphism $\Phi \in \operatorname{Diff}_{0}\left(\mathbb{R}^{d}\right)$. By Proposition 2 there exists a sequence $\left\{P_{n}\right\}_{n} \subset \mathrm{Gr}_{S} \mathcal{F}$ that tends to $\Phi$. So for $n$ sufficiently large last Proposition applies to $P_{n}^{-1} \circ \Phi$ and we have the following result.

Corollary 4. Let $\mathcal{F} \subseteq$ Vec $\mathbb{R}^{d}$ be a bracket generating family of vector fields and $N>0$ a positive integer. For every $\Phi \in \operatorname{Diff}_{0}\left(\mathbb{R}^{d}\right)$ there exists $P \in \mathrm{Gr}_{S} \mathcal{F}$ such that

$$
J_{0}^{N}(P)=J_{0}^{N}(\Phi)
$$




\section{Geometric statement of the main result}

The purpose of this section is to link the results of the last two sections in order to find an element in the group $\mathrm{Gr}_{S} \mathcal{F}$ with the same $N$-th jet of a given diffeomorphism and also close to it in the $C^{\infty}$-topology.

Proposition 5. Let $\mathcal{F} \subseteq V \mathbb{R}^{d}$ be a bracket generating family of vector fields. Let $N$ and $k$ be positive integers, $\varepsilon>0$, and $B$ ball of $\mathbb{R}^{d}$. For any $\Phi \in \operatorname{Diff}_{0}\left(\mathbb{R}^{d}\right)$, there exists $P \in \mathrm{Gr}_{S} \mathcal{F}$ such that

$$
J_{0}^{N}(P)=J_{0}^{N}(\Phi) \quad \text { and } \quad\|P-\Phi\|_{C^{k}(B)}<\varepsilon .
$$

Proof. We can suppose that $J_{0}^{N}(\Phi)=$ Id. Indeed, by Corollary 4 , there exists $Q \in \mathrm{Gr}_{S} \mathcal{F}$ such that $J_{0}^{N}(Q)=J_{0}^{N}(\Phi)$. Then we consider, instead of $\Phi$, the diffeomorphism $\Psi=\Phi \circ Q^{-1}$ which has trivial jet.

The idea of the proof is the same of Proposition 2. Since $J_{0}^{N}(\Phi)=\mathrm{Id}$, then $\Phi$ can be written as

$$
\Phi(x)=x+g(x),
$$

with $J_{0}^{N}(g)=0$. Consider the one parameter family of diffeomorphisms with trivial jet

$$
\Phi_{t}(x)=x+\operatorname{tg}(x) .
$$

This is a path in $\operatorname{Diff}\left(\mathbb{R}^{d}\right)$ from $\Phi_{0}=\operatorname{Id}$ to $\Phi_{1}=\Phi$. Let $V_{t}$ a nonautonomous vector field such that

$$
\Phi_{t}=\overrightarrow{\exp } \int_{0}^{t} V_{\tau} d \tau
$$

Let $X_{1}, \ldots, X_{d}$ be a frame of vector fields linearly independent at 0 such that $X_{i}=\operatorname{Ad} P^{i} f_{i}, P^{i} \in \operatorname{Gr} \mathcal{F}, f_{i} \in \mathcal{F}$. Therefore

$$
V_{t}=\sum_{i=1}^{d} a_{i}(t, \cdot) X_{i}
$$

with $a_{i}(t, \cdot) \in C^{\infty}\left(\mathbb{R}^{d}\right)$ for any $t \in[0,1]$. Note that, since $J_{0}^{N}\left(\Phi_{t}\right)=\operatorname{Id}$ and the vector fields $X_{i}$ are linearly independent, then $J_{0}^{N}\left(a_{i}(t, \cdot)\right)=0$ for any $t \in[0,1]$.

Now let $\mathcal{A}$ be the closed subspace of $C^{\infty}\left(\mathbb{R}^{d}\right)$ of smooth functions $\alpha$ such that $J_{0}^{N}(\alpha)=0$. By Proposition 1 there exists a piecewise constant in $t$ sequence $Z_{t}^{n} \in\left\{\alpha X_{i} \mid \alpha \in \mathcal{A}, i=1 \ldots, d\right\}$ such that

$$
\overrightarrow{\exp } \int_{0}^{t} Z_{\tau}^{n} d \tau \rightarrow \Phi_{t}, \quad \text { as } n \rightarrow \infty
$$


in the $C^{\infty}$-topology and uniformly with respect to $t \in[0,1]$.

So, if $P_{n}=\overrightarrow{\exp } \int_{0}^{1} Z_{\tau}^{n} d \tau$, then

$$
P_{n} \rightarrow \Phi, \quad \text { as } n \rightarrow \infty,
$$

in the standard topology. Now, for any $n$, we have that $P_{n} \in \mathrm{Gr}_{S} \mathcal{F}$ for the chain of equalities (6). Moreover $P_{n}$ has trivial jet. Indeed, since the sequence $Z_{t}^{n}$ is piecewise constant, then there exist intervals $I_{1}, \ldots, I_{h}$ such that

$$
Z_{t}^{n}=\alpha_{i} X_{j_{i}} \quad \text { for any } t \in I_{i},
$$

with $j_{i} \in\{1, \ldots, d\}$. So

$$
\begin{aligned}
J_{0}^{N}\left(P_{n}\right) & =J_{0}^{N}\left(\overrightarrow{\exp } \int_{0}^{1} Z_{t}^{n} d t\right) \\
& =J_{0}^{N}\left(e^{\left|I_{1}\right| \alpha_{1} X_{j_{1}}}\right) \circ \cdots \circ J_{0}^{N}\left(e^{\left|I_{h}\right| \alpha_{h} X_{j_{h}}}\right) \\
& =e^{\left|I_{1}\right| J_{0}^{N}\left(\alpha_{1}\right) J_{0}^{N}\left(X_{j_{1}}\right)} \circ \cdots \circ e^{\left|I_{h}\right| J_{0}^{N}\left(\alpha_{h}\right) J_{0}^{N}\left(X_{j_{h}}\right)} \\
& =\mathrm{Id},
\end{aligned}
$$

and the result is proved.

\section{$5 \quad$ Main Result}

In this last section we prove the main result using Proposition 5 and a fixed point argument. We start giving an equivalent formulation of Proposition 5 in terms of flows of the system:

$$
\dot{q}=\sum_{i=1}^{m} u_{i}(t, q) f_{i}(q), \quad q \in \mathbb{R}^{d} .
$$

Suppose that $\mathcal{F}=\left\{f_{1}, \ldots, f_{m}\right\}$ is a bracket generating family of vector field on $\mathbb{R}^{d}$. By Proposition 3 there exist smooth functions $a_{1}, \ldots, a_{k}$ such that

$$
J_{0}^{N}(\Phi)=J_{0}^{N}\left(e^{a_{1} f_{i_{1}}} \circ \cdots \circ e^{a_{k} f_{i_{k}}}\right),
$$

with $i_{j} \in\{1, \ldots, m\}$. Now there exist $m$ functions $u_{1}(t, q), \ldots, u_{m}(t, q)$ piecewise constant in $t$ such that

$$
J_{0}^{N}(\Phi)=J_{0}^{N}\left(\overrightarrow{\exp } \int_{0}^{1} \sum_{i=1}^{m} u_{i}(t, \cdot) f_{i} d t\right)
$$


We then proved the following Lemma.

Lemma 6. Let $\left\{f_{1}, f_{2}, \ldots, f_{m}\right\}$ be a bracket generating family of vector fields on $\mathbb{R}^{d}$. Consider the control system

$$
\dot{q}=\sum_{i=1}^{m} u_{i}(t, q) f_{i}(q), \quad q \in \mathbb{R}^{d},
$$

with controls $u_{i}$ piecewise constant with respect to $t \in[0,1]$ and smooth with respect to $q \in \mathbb{R}^{d}$, for every $i=1, \ldots, m$.

Let $N$ and $k$ be positive integers, $\varepsilon>0$, and $B$ ball in $\mathbb{R}^{d}$. For any $\Phi \in$ $\operatorname{Diff}_{0}\left(\mathbb{R}^{d}\right)$, there exist controls $u_{1}(t, q), \ldots, u_{m}(t, q)$ such that, if $P$ is the flow at time 1 of system (12), then

$$
J_{0}^{N}(P)=J_{0}^{N}(\Phi) \quad \text { and } \quad\|P-\Phi\|_{C^{k}(B)}<\varepsilon .
$$

What remains is to prove last result adding a drift $f_{0}$ to system (12). Moreover we want to have a certain regularity for the controls. Both these results can be proved with a fixed point argument. Indeed, let $\mathbf{U}$ the space of $m$-uples of controls $u(t, q)$ piecewise constant in $t$ and smooth with respect to $q$. Consider the map

$$
\begin{aligned}
& \tilde{F}: \quad \mathbf{U} \quad \longrightarrow \quad J_{0}^{N}\left(\operatorname{Diff}_{0}\left(\mathbb{R}^{d}\right)\right) \\
& \left(u_{1}, \ldots, u_{m}\right) \longmapsto J_{0}^{N}\left(\overrightarrow{\exp } \int_{0}^{1} \sum_{i=1}^{m} u_{i}(t, \cdot) X_{i} d t\right) \text {. }
\end{aligned}
$$

This map is continuous and, by last Lemma, it is also surjective. Moreover $\tilde{F}$ has a continuous right inverse. Indeed there is a smooth correspondence between the time-varying feedback controls $u_{1}, \ldots, u_{m}$ and the functions $a_{1}, \ldots, a_{k}$ in (10). By Implicit Function Theorem applied to map $F$ in (7), we have that the right inverse of $F$ is continuous and so is the right inverse of $\tilde{F}$.

In the next Lemma we prove, using a fixed point argument, that every small perturbation of a continuous surjective map with continuous right inverse and with finite dimensional target space is surjective too.

Lemma 7. Let $X$ be a topological space, $\varepsilon>0$, and $F: X \rightarrow \mathbb{R}^{n}$ a continuous and surjective with continuous right inverse. If $G: X \rightarrow \mathbb{R}^{n}$ is continuous and such that $\sup _{x \in K}|F(x)-G(x)|<\varepsilon$ for any $K \subseteq X$ compact, then $G$ is surjective. 
Proof. Let $F^{-1}$ be the right inverse of $F$ and define, for every $\bar{y}$ in $\mathbb{R}^{n}$, the map $\chi_{\bar{y}}(y)=y-G \circ F^{-1}(y)+\bar{y}$. Let $\delta=\varepsilon+\|\bar{y}\|$, then for every $y \in B_{\delta}=B_{\delta}(0)$ we have

$$
\begin{aligned}
\left\|\chi_{\bar{y}}(y)\right\| & \leq\left\|y-G \circ F^{-1}(y)\right\|+\|\bar{y}\| \\
& \leq \sup _{y \in B_{\delta}}\left\|y-G \circ F^{-1}(y)\right\|+\|\bar{y}\| \\
& \leq \sup _{x \in F^{-1}\left(B_{\delta}\right)}\|F(x)-G(x)\|+\|\bar{y}\| \\
& <\varepsilon+\|\bar{y}\| \\
& =\delta .
\end{aligned}
$$

So $\chi_{\bar{y}}\left(B_{\delta}\right) \subseteq B_{\delta}$ and, since the map $\chi_{\bar{y}}$ is continuous, by Brouwer Fixed Point Theorem, there exists $\tilde{y} \in B_{\delta}$ such that

$$
\chi_{\bar{y}}(\tilde{y})=\tilde{y},
$$

namely

$$
G \circ F^{-1}(\tilde{y})=\bar{y} .
$$

We proved that, for every $y \in \mathbb{R}^{n}$, there exists, $x \in X$ such that $y=G(x)$.

The Main Result can now be proved.

Theorem 8. Let $\left\{f_{1}, f_{2}, \ldots, f_{m}\right\}$ be a bracket generating family of vector fields on $\mathbb{R}^{d}$. Consider the control system

$$
\dot{q}=f_{0}(q)+\sum_{i=1}^{m} u_{i}(t, q) f_{i}(q), \quad q \in \mathbb{R}^{d},
$$

with controls $u_{i}$ such that:

(i) $u_{i}$ is polynomial with respect to $q \in \mathbb{R}^{d}$;

(ii) $u_{i}$ is a trigonometric polynomial with respect to $t \in[0,1]$;

for every $i=1, \ldots, m$.

Fix positive integers $N$ and $k, \varepsilon>0$, and $B$ ball of $\mathbb{R}^{d}$. For any $\Phi \in$ $\operatorname{Diff}_{0}\left(\mathbb{R}^{d}\right)$, there exist controls $u_{1}(t, q), \ldots, u_{m}(t, q)$ such that, if $P$ is the flow at time 1 of system (14), then

$$
J_{0}^{N}(P)=J_{0}^{N}(\Phi) \quad \text { and } \quad\|P-\Phi\|_{C^{k}(B)}<\varepsilon .
$$


Proof. Proof splits into three steps. First prove that it is sufficient to consider controls that are polynomials with respect to $q \in \mathbb{R}^{d}$, then we add the drift to the system, and finally we find controls that are trigonometric polynomials with respect to $t$ by smoothing the time dependence of the piecewise constant controls. Let us start with the first step and note that, as a consequence of the density of polynomials in the space of smooth functions on a bounded set and bu Lemma 7 , we can assume that $u_{i}(t, q)$ is a polynomial in $q$ for every $t \in[0,1]$ and for every $i=1, \ldots, m$.

Now set $\mathbf{Y}=J_{0}^{N}\left(\operatorname{Diff}_{0}\left(\mathbb{R}^{d}\right)\right)$ and consider the family of continuous maps

$$
\begin{aligned}
& F_{\varrho}: \quad \mathbf{U} \quad \longrightarrow \quad \mathbf{Y} \\
& \left(u_{1}, \ldots, u_{m}\right) \longmapsto J_{0}^{N}\left(\overrightarrow{\exp } \int_{0}^{\varrho} \varrho f_{0}+\sum_{i=1}^{m} u_{i}(t, \cdot) X_{i} d t\right) .
\end{aligned}
$$

We claim that, if there exists $\varrho>0$ such that $F_{\varrho}$ is surjective then so is $F_{\varrho}$ for $\varrho=1$. Indeed

$$
F_{\varrho}\left(u_{1}(t, \cdot), \ldots, u_{m}(t, \cdot)\right)=F_{1}\left(\frac{u_{1}(t / \varrho, \cdot)}{\varrho}, \ldots, \frac{u_{m}(t / \varrho, \cdot)}{\varrho}\right) .
$$

Similarly the map $\tilde{F}_{\varrho}\left(u_{1}, \ldots, u_{m}\right)=J_{0}^{N}\left(\overrightarrow{\exp } \int_{0}^{\varrho} \sum_{i=1}^{m} u_{i}(t, \cdot) X_{i} d t\right)$ is surjective for every $\varrho>0$ since it is equal to the map $\tilde{F}$ defined in (13) up to rescalings of the time dependence of the controls $u_{i}$. We have, for small $\varrho>0$, that $F_{\varrho}$ is a small perturbation of $\tilde{F}_{\varrho}$. So Lemma 7 applies and $F_{1}$ is surjective.

Finally, for any control $u(t, q)$ piecewise constant in $t$ and polynomial in $q$, we can write

$$
u(t, q)=\sum_{|\alpha|=0}^{N} a_{\alpha}(t) q^{\alpha},
$$

with $\alpha$ multi-index and $a_{\alpha}(t)$ piecewise constant. For every $\alpha$, the function $a_{\alpha}$ admits a Fourier expansion of the form

$$
a_{\alpha}(t)=\sum_{j=0}^{\infty} \eta_{\alpha}^{j} \cos (2 \pi j t)+\xi_{\alpha}^{j} \sin (2 \pi j t) .
$$

Consider the trigonometric polynomial

$$
a_{\alpha}^{n}(t)=\sum_{j=0}^{n} \eta_{\alpha}^{j} \cos (2 \pi j t)+\xi_{\alpha}^{j} \sin (2 \pi j t),
$$


then $a_{\alpha}^{n}(t) \rightarrow a_{\alpha}(t)$ as $n \rightarrow \infty$ in $L^{1}[0,1]$. So let

$$
u^{n}(t, q)=\sum_{|\alpha|=0}^{N} a_{\alpha}^{n}(t) q^{\alpha},
$$

then

$$
u^{n}(t, q) \rightarrow u(t, q), \quad \text { as } n \rightarrow \infty,
$$

and the convergence is uniform with all derivatives on compact sets of $\mathbb{R}^{d}$ and in $L^{1}[0,1]$ with respect to $t$.

Let $G_{n}$ be the family of continuous maps

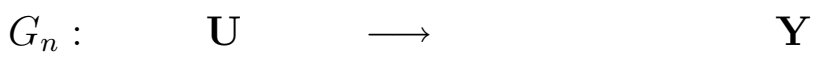

$$
\begin{aligned}
& \left(u_{1}, \ldots, u_{m}\right) \longmapsto J_{0}^{N}\left(\overrightarrow{\exp } \int_{0}^{1} f_{0}+\sum_{i=1}^{m} u_{i}^{n}(t, \cdot) X_{i} d t\right) .
\end{aligned}
$$

By the convergence in (15), $G_{n} \rightarrow F_{1}$ as $n \rightarrow \infty$ for every $\left(u_{1}, \ldots, u_{m}\right) \in \mathbf{U}$, then there exists $n_{0}$ integer for which Lemma 7 applies. Therefore the map $G_{n_{0}}$ is surjective and Theorem follows.

Remark 1. Clearly the statement of Theorem 8 holds also if we consider the jet at a point $q \in \mathbb{R}^{d}$. It is also clear that it is possible to fix a finite number of points in $\mathbb{R}^{d}$, say $q_{1}, \ldots, q_{\ell}$, and find an admissible diffeomorphism, arbitrary close to a given one, that realize its $N$-th jet at all the points $q_{1}, \ldots, q_{\ell}$ at the same time.

\section{Appendix}

Here we prove Proposition 1. The proof is based on the following well-known fact (see, for instance, [1, Lemma 8.2]).

Lemma 9. Let $Z_{t}$ and $Z_{t}^{n}$ for $n=1,2, \ldots$ and $t \in[0,1]$ be nonautonomous vector fields on $M$. If

$$
\int_{0}^{t} Z_{\tau}^{n} d \tau \rightarrow \int_{0}^{t} Z_{\tau} d \tau, \quad \text { as } n \rightarrow \infty
$$

in the standard $C^{\infty}$ topology and uniformly with respect to $t \in[0,1]$, then

$$
\overrightarrow{\exp } \int_{0}^{t} Z_{\tau}^{n} d \tau \rightarrow \overrightarrow{\exp } \int_{0}^{t} Z_{\tau} d \tau, \quad \text { as } n \rightarrow \infty
$$

in the same topology. 
Proof of Proposition 1. First, note that we can suppose, without loss of generality, that $a_{i}(t, \cdot)$ is piecewise constant in $t$ for every $i=1, \ldots, k$. Indeed, for any $i=1, \ldots, k$, the sequence

$$
a_{i}^{n}(t, q)=n \sum_{j=1}^{n} \int_{\frac{j-1}{n}}^{\frac{j}{n}} a_{i}(\tau, q) d \tau \chi_{j}^{n}(t)
$$

where $\chi_{j}^{n}(t)$ is the characteristic function of the interval $\left[\frac{j-1}{n}, \frac{j}{n}\right]$, is such that

$$
\int_{0}^{t} \sum_{i=1}^{k} a_{i}^{n}(\tau, \cdot) X_{i} d \tau \rightarrow \int_{0}^{t} V_{\tau} d \tau, \quad \text { as } n \rightarrow \infty,
$$

uniformly with respect to $t$ and in the $C^{\infty}$-topology. Therefore Lemma 9 allows us to suppose that $a_{i}(t, \cdot)$ is piecewise constant in $t$ for every $i$.

Let $\ell$ be a positive integer such that $V_{t}$ is constant on $\left[\frac{j-1}{\ell}, \frac{j}{\ell}\right]$ for every $j=1, \ldots, \ell$. We can write

$$
a_{i}(t, q)=\sum_{j=1}^{\ell} a_{i}^{j}(q) \chi_{j}^{\ell}(t)
$$

with $a_{i}^{j}(q) \geq 0$ for every $q \in \mathbb{R}^{d}$. Let

$$
\alpha^{j}=\sum_{i=1}^{k} a_{i}^{j}
$$

and let $\left\{\varepsilon_{n}\right\}$ a sequence of nonnegative smooth functions of $\mathbb{R}^{d}$ such that $\varepsilon_{n}(0)=0$ for every $n$ and $\varepsilon_{n} \rightarrow 0$ as $n \rightarrow \infty$ in the $C^{\infty}$-topology. Then $\alpha_{n}^{j}=\alpha^{j}+\varepsilon_{n}$ is strictly positive on $\mathbb{R}^{d} \backslash\{0\}$ for every $j$ and $n$.

Now, for every positive integer $n$ and $j=1, \ldots, \ell$, let $b_{n}^{j, i}=a_{i}^{j} / \alpha_{n}^{j}$ and consider the family of intervals:

$$
A_{n}^{j, i}=\bigcup_{m=0}^{n-1}\left[\frac{j-1}{\ell}+\frac{m}{n \ell}+\frac{b_{n}^{j, 1}+\cdots+b_{n}^{j, i-1}}{n \ell}, \frac{j-1}{\ell}+\frac{m}{n \ell}+\frac{b_{n}^{j, 1}+\cdots+b_{n}^{j, i}}{n \ell}\right),
$$

for $i=2, \ldots, k$, and

$$
A_{n}^{j, 1}=\bigcup_{m=0}^{n-1}\left[\frac{j-1}{\ell}+\frac{m}{n \ell}, \frac{j-1}{\ell}+\frac{m}{n \ell}+\frac{b_{n}^{j, 1}}{n \ell}\right) .
$$


The sequence of vector fields

$$
Z_{t}^{n}=\alpha_{n}^{j} X_{i}, \quad \text { if } t \in A_{n}^{j, i},
$$

is such that

$$
\int_{0}^{t} Z_{\tau}^{n} d \tau \rightarrow \int_{0}^{t} V_{\tau} d \tau, \quad \text { as } n \rightarrow \infty .
$$

In the standard topology and uniformly with respect to $t \in[0,1]$. The statement then follows from Lemma 9.

\section{References}

[1] A. A. Agrachev, Yu. L. Sachkov, Control theory from the geometric viewpoint. Springer-Verlag, Berlin, 2004.

[2] R. W. Brockett, Asymptotic stability and feedback stabilization. In: Differential Geometric Control Theory (R. W. Brockett, R. S. Millman, H. J. Sussmann, eds.), Birkhaäuser, Basel, 1983.

[3] W.-L. Chow, Über systeme von linearen partiellen differentialgleichungen erster ordinung. Math. Ann., 1939, v.117, 98-105.

[4] J.-M. Coron, Global asymptotic stabilization for controllable systems without drift. Math. Control Signals Systems, 1992, v.5, 295-312.

[5] J.-M. Coron, On the stabilization in finite time of locally controllable systems by means of continuous time-varying feedback law. SIAM J. Control Optim. 1995, v.33, 804-833.

[6] J.-M. Coron, Control and nonlinearity. Mathematical Surveys and Monographs, 136. American Mathematical Society, Providence, RI, 2007. xiv +426 pp.

[7] R. V. Gamkrelidze, Principles of optimal control. Plenum Press, New York, 1978.

[8] P. K. Rashevski, About connecting two points of complete nonholonomic space by admissible curve. Uch Zapiski Ped. Inst. Libknexta 2, 1938, 8394. 
[9] H. J. Sussmann, Orbits of families of vector fields and integrability of distributions. Trans. Amer. Math. Soc., 1973, v.180, 171-188. 